\title{
INDECOMPOSABLE COMPACT PERTURBATIONS OF THE BILATERAL SHIFT
}

\author{
DOMINGO A. HERRERO
}

\begin{abstract}
Recent results of M. Radjabalipour and H. Radjavi assert that the sum of a normal operator $N$ with spectrum on a smooth Jordan curve and a compact operator $K$ in the Macaev ideal $\mathfrak{S}_{\omega}$ is decomposable provided the spectrum of $N+K$ does not fill the interior of the curve. Examples are given to show that this result cannot be essentially improved by taking $K$ in a larger ideal.
\end{abstract}

1. Let $\mathcal{E}(\mathcal{H})$ be the algebra of all (bounded linear) operators on a complex Hilbert space $\mathcal{H}$. The Macaev ideal $\mathfrak{S}_{\omega}$ is the set of all compact operators $K$ in $\mathcal{L}(\mathcal{K})$ such that $\Sigma_{1}^{\infty} \mu(n) / n<\infty$, where $\mu(1), \mu(2), \ldots$, are the eigenvalues of $\left(K^{*} K\right)^{1 / 2}$ arranged in decreasing order and repeated according to multiplicity.

Compact perturbations of normal operators with spectrum on a smooth Jordan curve by an operator $K \in \mathfrak{S}_{\omega}$ are known to have a rich family of invariant subspaces. To make this precise, several definitions will be necessary:

An invariant subspace $\mathscr{T}$ of $T \in \mathcal{L}(\mathcal{H})$ is a maximal spectral subspace of $T$ if $\mathscr{\Re} \mathfrak{N}$ for all invariant subspaces $\Re$ of $T$ such that the spectrum $\Lambda(T \mid \mathcal{\Re})$ of the restriction of $T$ to $\Re$ is contained in $\Lambda(T \mid \Re) . T$ is decomposable (in the sense of [1]) if for every finite covering $G_{j}, j=1$, $2, \ldots, n$, of $\Lambda(T)$ there exists a set of maximal spectral subspaces $\mathscr{K}_{j}, j=1$, $2, \ldots, n$, of $T$ such that $\Lambda\left(T \mid \mathcal{F}_{j}\right) \subset G_{j}, j=1,2, \ldots, n$, and $\mathcal{K}=\mathcal{K}_{1}+\mathcal{K}_{2}$ $+\cdots+\mathcal{H}_{n}$. Moreover, $T$ is called strongly decomposable if $T \mid \mathcal{T}$ is decomposable for every maximal spectral subspace $\mathfrak{R}$.

M. Radjabalipour and H. Radjavi [10]-[13] have improved a result of V. I. Macaev about compact perturbations of hermitian operators [9] by proving the following:

(i) Let $T$ be the sum of an operator $A$ having spectum on a $C^{2}$ Jordan curve $J$ and a compact operator $K \in \Im_{\omega}$. Assume that $\left\|(z-A)^{-1}\right\| \leqslant$ $C / d(z)$, where $d(z)$ is the distance from $z$ to $\Lambda(A)$ and $C \geqslant 1$ is a constant

Received by the editors April 2, 1975 and, in revised form, June 5, 1975, October 21, 1975 and May 24, 1976.

AMS (MOS) subject classifications (1970). Primary 47A15, 47A55.

Key words and phrases. Bilateral weighted shifts, decomposable operators, Macaev ideal of compact operators. 
independent of $z$, and that $\Lambda(T)$ does not fill the interior of $J$. Then $T$ is strongly decomposable.

(ii) Assume that $\Lambda(T)$ is contained in a $C^{2}$ Jordan curve $J$ and that there exist a positive number $\varepsilon$ and a nonincreasing function $M:(0, \varepsilon) \rightarrow(0, \infty)$ such that $\int_{0}^{\varepsilon} \log ^{(2)} M(t) d t<\infty\left(\log ^{(m)} x\right.$ denotes the $m$ th iterated logarithm). If $\left\|(z-T)^{-1}\right\| \leqslant M[d(z)]$ for $z \notin J$, then $T$ is strongly decomposable. This is true, in particular, if $M(t)=\exp \left(\exp t^{-p}\right), 0<p<1$.

It will be shown that these two results are essentially sharp by proving that compact perturbations of the (unitary!) bilateral shift $U$ in $l^{2}$ (defined by $U e_{n}=e_{n+1}$ for all $n$ in the set $\mathbf{Z}$ of all integers, where $\left\{e_{n}\right\}$ is the canonical basis of $\ell^{2}$ ) by an operator in a certain class of ideals which are only "slightly larger" than $\mathfrak{S}_{\omega}$ fail to be decomposable.

The author wishes to thank Professor Mehdi Radjabalipour for making available his unpublished papers and the referee for pointing out a mistake in the proof of part (a) of the theorem and for providing a suggestion which made it possible to simplify the proof of part (c).

2. Let $\left\{a_{n}\right\}$ be a bounded two-sided sequence of positive reals and define the bilateral weighted shift $B$ in $\ell^{2}$ by $B e_{n}=a_{n} e_{n+1}, n \in \mathbf{Z}$. If $\lim _{n \rightarrow \pm \infty} a_{n}=1$, then $B-U$ is compact and $\Lambda(B)$ is the boundary $\partial D$ of the unit disc $D=\{z:|z|<1\}$.

Define $w_{0}=1, w_{n}=a_{0}, a_{1} \cdots a_{n-1}$ and $w_{-n}=\left(a_{-1} a_{-2} \cdots a_{-n}\right)^{-1}$ for all positive $n$. Then $B$ is unitarily equivalent to multiplication by $z$ on the space

$$
L^{2}\left(\mathrm{w}=\left\{w_{n}\right\}\right)=\left\{f(z)=\sum_{n \in Z} b_{n} z^{n}:\|f\|^{2}=\sum\left|b_{n} w_{n}\right|^{2}<\infty\right\}
$$

of formal Laurent series [3], [6]. Now we are in a position to construct the counterexamples.

THEOREM. Let $\varphi=\left\{\varphi_{n}\right\}_{1}^{\infty}$ be a nonincreasing sequence of positive reals and assume that: (i) $\left\{\varphi_{n} \log e n\right\}$ is nonincreasing, (ii) $n\left(\varphi_{n}-\varphi_{n+1}\right) \rightarrow 0$, and (iii) $\Sigma \varphi_{n} / n=\infty$. Let $B$ be the bilateral weighted shift defined by $B e_{n}=a_{n} e_{n+1}$, where $a_{n}=w_{n+1} / w_{n}, w_{0}=1$ and $w_{n}=w_{-n}=\exp \left(n \varphi_{n}\right)$ for $n=1,2, \ldots$ Then

(a) Every nonzero invariant subspace $\Re$ of $B$ is either invariant under $B^{-1}$ and satisfies $\Lambda(B \mid \Re)=\partial D=\Lambda(B)$, or it is not invariant under $B^{-1}$ and $\Lambda(B \mid \mathfrak{T})=D^{-}$is the closed unit disc.

(b) $K=B-U$ is a compact operator such that the eigenvalues of $\left(K^{*} K\right)^{1 / 2}$ (ordered as above indicated) satisfy $\mu_{2 n-1}, \mu_{2 n} \leqslant C \varphi_{n}$, where $C$ is a constant independent of $n$.

(c) $\log ^{(2)}\left\|(z-B)^{-1}\right\| \leqslant M / d(z)$, for a suitable constant $M$.

Proof. (a) This will follow by using the same kind of "sandwich theory" as in [4]: 
Let $L^{1}(\mathbf{w})=\left\{f(z)=\sum b_{n} z^{n}:\|f\|_{1}+\sum\left|b_{n} w_{n}\right|<\infty\right\}$ and let $L^{1}(\sqrt{ } \mathbf{w})$ be similarly defined, with $w_{n}$ replaced by $\vee w_{n}, n \in \mathbf{Z}$ (the norm of an element of $L^{1}(\sqrt{ } w)$ will be denoted by $\left.\|\cdot\|^{\prime \prime}\right)$. Then, for every finite $\operatorname{sum} f(z)=\Sigma b_{n} w_{n}$, by the Cauchy-Schwarz inequality we have

$$
\begin{aligned}
\|f\|^{\prime \prime} & =\sum\left|b_{n} \vee w_{n}\right|=\sum\left|b_{n} w_{n}\right| / \vee w_{n} \\
& \leqslant\left(\sum\left|b_{n} w_{n}\right|^{2}\right)^{1 / 2}\left(\sum 1 / w_{n}\right)^{1 / 2}=C\|f\|,
\end{aligned}
$$

where $C=\left(\Sigma 1 / w_{n}\right)^{1 / 2}<\infty$ is a constant independent of $f$. It readily follows that $(1 / C)\|f\|^{\prime \prime} \leqslant\|f\| \leqslant\|f\|_{1}$ and, therefore, that $L^{1}(\mathbf{w}) \subset L^{2}(w) \subset L^{1}(\sqrt{ } w)$ and both inclusions are continuous.

It follows from (i) and (ii) that $\lim _{n \rightarrow \pm \infty} a_{n}=1$ and, therefore, $B$ is a compact perturbation of $U$ and $\Lambda(B)=\partial D$ (see [3], [4], [6]). Moreover, it follows from (i)-(iii) and the results of [2], [4] that $L^{1}(w)$ and $L^{1}(\sqrt{ } w)$ are actually algebras (under pointwise multiplication) of quasi-analytic functions defined on $\partial D$. I.e., $L^{1}(\sqrt{ } \mathbf{w})$ is contained in $C^{\infty}(\partial D)$; if $f, g \in L^{1}(w)$ $\left(L^{1}(\sqrt{ } \mathbf{w})\right)$, then $f(z) \cdot g(z) \in L^{1}(\mathbf{w})\left(L^{1}(\sqrt{ } \mathbf{w})\right.$, resp.) and the vanishing of $f(z) \in L^{1}(\sqrt[V]{ } \mathbf{w})$ together with all its derivatives at some point $\lambda \in \partial D$ implies that $b_{n}=0$ for all $n$.

Let $\mathfrak{N}$ be a nonzero invariant subspace of $B$. If $\mathfrak{N}$ is not invariant under $B^{-1}$, then $0 \in \Lambda(B \mid \Re)$ and it follows that $\Lambda(B \mid \Re)=D^{-}$; if $\Re$ is also invariant under $B^{-1}$, then $\Lambda(B \mid \Re) \subset \partial D$ (these two results easily follow from [7], [8]).

In the second case, the closure $\Re$ of $\mathscr{T}$ in $L^{1}(\sqrt{ } w)$ is an ideal of this algebra and we can proceed as in [4] in order to show that

$$
\Re=\bigcap_{j=1}^{m}\left\{f \in L^{1}(\sqrt{ } \mathbf{w}): f\left(z_{j}\right)=f^{\prime}\left(z_{j}\right)=\cdots=f^{\left(m_{j}-1\right)}\left(z_{j}\right)=0\right\}
$$

for a finite set of points $z_{j} \in \partial D, j=1,2, \ldots, m$, and positive integers $m_{j}$, $j=1,2, \ldots, m$, so that $\operatorname{dim} L^{1}(\sqrt{ } \mathbf{w}) / \mathscr{C}=n=\sum_{j=1}^{m} m_{j}<\infty$. Clearly, $\mathfrak{N}$ $\subset \Re \cap L^{2}(\mathbf{w})$ and for every $\lambda \in \partial D \backslash\left\{z_{1}, z_{2}, \ldots, z_{m}\right\}$ there exists a function $h_{\lambda} \in \mathfrak{N}$ such that $h_{\lambda}(\lambda) \neq 0$.

Let $\Re_{0}\left(\Re^{\prime \prime}\right)$ be the closure of $(B-\lambda) \Re$ in $L^{2}(w)$ (in $L^{1}(\sqrt{ } w)$, resp.). Since $(B-\lambda) f(z)=(z-\lambda) f(z)$, it readily follows that $\Re^{\prime \prime}=\Re \cap\{f \in$ $\left.L^{1}(\sqrt{ } w): f(\lambda)=0\right\}$ has codimension 1 in $\Re$. In particular, $h_{\lambda} \in \mathscr{N} \backslash \mathscr{K}$ ", a fortiori, $h_{\lambda} \notin \Re_{0}\left(\Re_{0} \subset \Re \cap \Re^{\prime \prime}\right)$ and, therefore, $(B-\lambda) \Re$ is not dense in $\mathfrak{N}$. Hence, $\lambda \in \Lambda(B \mid \mathscr{N})$.

Since this holds for all but finitely many $\lambda$ 's in $\partial D$, it is not difficult to conclude that $\partial D \subset \Lambda(B \mid \Re)$ and, therefore, $\Lambda(B \mid \mathscr{N})=\partial D$, as promised.

If $\Re$ contains a nonzero function $L^{1}(w)$, it is easy to see that $\Re \cap L^{1}(w)$ is actually an ideal of finite codimension $n$ in $L^{1}(w)$ and, a fortiori, that $\Re$ also has codimension $n$ in $L^{2}(w)$. (The details are left to the reader. The author conjectured that $\Re \cap L^{1}(w) \neq\{0\}$ for every invariant subspace $\Re$, but was unable to prove it.) 
(b) This follows immediately from $K e_{n}=(B-U) e_{n}=\left(a_{n}-1\right) e_{n+1}$ (see [5]).

(c) Since $U$ is normal, $\gamma(t)=\max \left\{\left\|(z-U)^{-1}\right\|: d(z)=t\right\}=1 / t$. On the other hand (b) and condition (i) show that $\mu_{n} \leqslant k / \log e n$, for some constant $k>0$ and for all $n=1,2, \ldots$, and, therefore, $\nu(t)=\max \left\{n: \mu_{n}>1 / t\right\} \leqslant$ $e^{k t}$. Now we are in a position to apply Theorem 1 of [13], whence the result follows.

3. The sequences

$$
\left\{\varphi_{n}=\varphi_{n}(m)=\left[\log M_{1} n \cdot \log ^{(2)} M_{2} n \cdots \cdot \log ^{(m)} M_{m} n\right]^{-1}\right\},
$$

where $M_{1}=e$ and $M_{k+1}=\exp \left(M_{k}\right)$ for $k=1,2, \ldots, m-1 \quad(m=1$, $2, \ldots)$, are concrete examples of sequences satisfying conditions (i)-(iii). Moreover, if $m_{n}>\infty$ and

$$
\varphi_{n}=\psi_{n}\left(m_{n}\right)=\varphi_{n}\left(m_{n}\right) /\left[\log \left(m_{n}+1\right) M_{m_{n}+1} n\right]^{2}
$$

satisfies (iii), then it actually satisfies (i)-(iii).

Given an arbitrary increasing function $h(n)$ such that $h(1)=1$ and $\lim _{n \rightarrow \infty} h(n)=\infty$, it is not difficult to find a sequence $\left\{m_{n}\right\}_{n=1}^{\infty}$ such that $\left\{\psi_{n}\left(m_{n}\right)\right\}$ satisfies (i)-(iii), but $\Sigma_{1}^{\infty} \psi_{n}\left(m_{n}\right) /[n h(n)]<\infty$. Now we can give a very precise meaning to our introductory sentence "these two results are essentially sharp":

COROLlARY. Let $h(n)$ be an arbitrary increasing function such that $h(1)=1$ and $\lim _{n \rightarrow \infty} h(n)=\infty$, and let $\mathfrak{\subseteq}_{h}=\left\{K \in \mathcal{L}\left(\ell^{2}\right): K\right.$ is compact and $\left.\sum \mu(n) /[n h(n)]<\infty\right\}$. Then there exists an operator $K$ in the ideal $\mathfrak{S}_{h}$ such that $U+K$ is indecomposable, even when its resolvent satisfies the growth conditions of the Theorem.

The proof follows immediately from the Theorem and the above observations.

4. An example. Let $\varphi_{n}=\left(\log e n \cdot \log ^{(2)} e^{2} n\right)^{-1}$; then a straightforward computation shows that $\mu_{n}$ is of the same order of magnitude as $\varphi_{n}(n=1,2, \ldots)$ and that, in this case, $\nu(t) \leqslant \exp (k t / \log t)$, whence (by applying once again Theorem 1 of [13]) we can improve the estimation (c) of the Theorem to

$$
\log ^{(2)}\left\|(z-B)^{-1}\right\| \leqslant M /[d(z) \cdot \log 1 / d(z)],
$$

for a suitable constant $M$ and for $0<d(z)<\frac{1}{2}$.

\section{REFERENCES}

1. I. Colojară and C. Foias, Theory of generalized spectral operators, Gordon and Breach, New York, 1968.

2. Y. Domar, Harmonic analysis based on certain commutative Banach algebras, Acta Math. 96 (1956), 1-66. MR 17, 1228.

3. R. Gellar, Operators commuting with a weighted shift, Proc. Amer. Math. Soc. 23 (1969), 538-545. MR 41 \#4277a. 
4. R. Gellar and D. A. Herrero, Hyperinvariant subspaces of bilateral weighted shifts, Indiana Univ. Math. J. 23 (1973/74), 771-790. MR 48 \# 9417.

5. I. C. Gohberg and M. G. Krein, Introduction to the theory of linear nonselfadjoint operators, "Nauka", Moscow, 1965; English transl, Transl. Math. Monographs, vol. 18, Amer. Math. Soc., Providence, R.I., 1969. MR 36 \#3137; 39 \# 7447.

6. P. R. Halmos, A Hilbert space problem book, Van Nostrand, Princeton, N. J., 1967. MR 34 \#8178.

7. D. A. Herrero, On analytically invariant subspaces and spectra, Trans. Amer. Math. Soc. (to appear).

8. D. A. Herrero and N. Salinas, Analytically invariant and bi-invariant subspaces, Trans. Amer. Math. Soc. 173 (1972), 117-136. MR 47 \#856.

9. V. I. Macaev, On a class of completely continuous operators, Dokl. Akad. Nauk SSSR 139 (1961), 548-551 = Soviet Math. Dokl. 2 (1961), 972-975. MR 24 \# A1617.

10. M. Radjabalipour, Growth conditions and decomposable operators, Canad. J. Math. 26 (1974), 1372-1379.

11. On decomposability of compact perturbations of operators, Proc. Amer. Math. Soc. 53 (1975), 159-164.

12. M. Radjabalipour and H. Radjavi, On decomposability of compact perturbations of normal operators, Canad. J. Math. 27 (1975), 725-735.

13. , On invariant subspaces of compact perturbations of operators (to appear).

Departmento de Matemáticas, Universidad Simón Bolívar, Caracas, Venezuela

Current address: Departamento de Matemáticas, Instituto Venezolano de Investigaciones Cientificas, Caracas, Venezuela 